\title{
Cooperative Diversity for Intervehicular Communication: Performance Analysis and Optimization
}

\author{
Hacı İlhan, Student Member, IEEE, Murat Uysal, Senior Member, IEEE, and İbrahim Altunbaş
}

\begin{abstract}
Although there has been a growing literature on cooperative diversity, the current literature is mainly limited to the Rayleigh fading channel model, which typically assumes a wireless communication scenario with a stationary base station antenna above rooftop level and a mobile station at street level. In this paper, we investigate cooperative diversity for intervehicular communication based on cascaded Nakagami fading. This channel model provides a realistic description of an intervehicular channel where two or more independent Nakagami fading processes are assumed to be generated by independent groups of scatterers around the two mobile terminals. We investigate the performance of amplify-and-forward relaying for an intervehicular cooperative scheme assisted by either a roadside access point or another vehicle that acts as a relay. Our diversity analysis reveals that the cooperative scheme is able to extract the full distributed spatial diversity. We further formulate a power-allocation problem for the considered scheme to optimize the power allocated to the broadcasting and relaying phases. Performance gains up to $3 \mathrm{~dB}$ are obtained through optimum power allocation, depending on the relay location.
\end{abstract}

Index Terms-Cooperative diversity, fading channels, intervehicular communication, relay-assisted transmission.

\section{INTRODUCTION}

$\mathbf{I}$ NTERVEHICULAR communication is an integral part of intelligent transportation systems (ITSs), which have been receiving growing attention in recent years [1], [2]. The concept of ITS has mainly been originated to advance transportation safety and efficiency through dissemination of road and traffic information, e.g., real-time updates regarding collisions, incidents, congestion, surface, and weather conditions, and

Manuscript received May 5, 2008; revised November 5, 2008. First published February 6, 2009; current version published August 14, 2009. This paper was presented in part at the IEEE Vehicular Technology Conference (VTC-Spring) [24], Singapore, May 2008, and the IEEE Vehicular Technology Conference (VTC-Fall) [25], Calgary, AB, Canada, September 2008. The work of H. İlhan and İ. Altunbaş was supported by the Scientific and Technological Research Council of Turkey under Project 107E022. The work of M. Uysal was supported in part by the Natural Sciences and Engineering Research Council under Collaborative Research and Development Grant CRDPJ348999-06. The review of this paper was coordinated by Prof. W. Su.

H. İlhan and I. Altunbas are with the Department of Electronics and Communication Engineering, Istanbul Technical University, 34469 Istanbul, Turkey (e-mail: ilhanh@itu.edu.tr; ibraltunbas@itu.edu.tr).

M. Uysal is with the Department of Electrical and Computer Engineering, University of Waterloo, Waterloo, ON N2L3G1, Canada (e-mail: muysal@ece. uwaterloo.ca).

Digital Object Identifier 10.1109/TVT.2009.2014685 coordination of vehicles at critical points such as blind crossings and highway entries. A variety of broadband in-vehicle applications (such as high-speed Internet access from within the vehicular network, cooperative downloading, network gaming among passengers of adjacent cars, and virtual meetings among coworkers) is also envisioned [1]-[6] as a result of everincreasing dependence on the Internet.

Although there has been a growing literature on the networking and application layers in vehicular networks, the relevant literature on the physical-layer aspects is sparse. The main challenge facing the deployment of vehicular ad hoc networks (VANETs) indeed manifests itself as their main advantage, i.e., the lack of infrastructure. This makes cooperative diversity (also known as user cooperation or cooperative communication) [7]-[9] an ideal physical-layer solution for VANETs. Cooperative diversity exploits the broadcast nature of wireless transmission, i.e., the cost-free possibility of the transmitted signals being received by other than the destination node, and thus, a source node can get help from other nodes by relaying the information message to the destination node. The source and its relays effectively form a virtual antenna array to exploit spatial diversity advantages.

Cooperative diversity has extensively been investigated in the literature [7]-[12]; however, the current results are mainly limited to the Rayleigh fading channel model, which is commonly used to characterize the cellular radio systems. This model typically assumes a wireless communication scenario with a stationary base station antenna above rooftop level and a mobile station at street level. On the other hand, in intervehicular communication systems, both the transmitter and receiver antennas are in motion, and their antennas are relatively at lower elevations, invalidating the Rayleigh fading assumption. Various experimental results and theoretical analysis (see, e.g., [13], [14], and the references therein) demonstrate that the Rayleigh channel model and the related second-order channel statistics originally proposed for a base station-to-mobile link fail to provide an accurate model for dynamic mobile-to-mobile link. Instead, the cascaded (double) Rayleigh fading channel model has been proposed [14], [15], which provides a realistic description of an intervehicular channel where two Rayleigh fading processes are assumed to be generated by independent groups of scatterers around the two mobile terminals. A generalized channel model, i.e., the so-called $N^{*}$ Nakagami, has further been proposed in [16], which involves the product of $N$ Nakagami- $m$ distributed random variables. 
In this paper, we investigate cooperative diversity in the context of vehicular communication. We consider a vehicle-tovehicle $(\mathrm{V} 2 \mathrm{~V})$ scenario under two different scenarios. In the first scenario, the source vehicle is assisted by another vehicle in its vicinity. All underlying channels are modeled as cascaded Nakagami fading (assuming $N=2$ ). ${ }^{1}$ In the second scenario, a roadside access point (AP) acts as a relay. Therefore, the channel between source and destination vehicles is modeled by cascaded Nakagami fading, whereas source-to-relay AP and relay AP-to-destination channels are modeled by Nakagami fading. We consider the receive diversity protocol of [7] with amplify-and-forward relaying. We first obtain the diversity order for these two relay-assisted vehicular scenarios through the derivation of the pairwise error probability (PEP). Then, building upon a union bound on the bit error rate (BER), we formulate a power-allocation problem to determine how the overall transmit power should be shared between broadcasting and relaying phases for performance optimization.

The rest of this paper is organized as follows: In Section II, we introduce the relay-assisted $\mathrm{V} 2 \mathrm{~V}$ transmission model. In Section III, we derive the PEP expressions for two scenarios under consideration and discuss the diversity order. In Section IV, we present union bounds on the BER, which are used as objective functions for optimization, optimization procedure, and results of optimization. In Section V, we provide Monte Carlo simulation results to demonstrate the error rate performance of the relay-assisted $\mathrm{V} 2 \mathrm{~V}$ scheme for various relay locations and compare the performance of equal power allocation (EPA) and optimum power allocation (OPA) schemes. We finally conclude in Section VI.

\section{Channel and Transmission Model}

We consider a single-relay scenario in which source, relay, and destination nodes operate in half-duplex mode and are equipped with a single pair of transmit and receive antennas. We study two different scenarios based on the relay type.

In the first scenario [see Fig. 1(a)], the source vehicle is assisted by another vehicle, whereas in the second scenario [see Fig. 1(b)], a roadside AP acts a relay. We assume an aggregate channel model that takes into account both the long-term path loss and short-term fading. This allows us to explicitly consider the effects of the relay location in our transmission model. In Fig. 1(a) and (b), $d_{S D}, d_{S R}$, and $d_{R D}$ are the distances of source-to-destination $(S \rightarrow D)$, source-to-relay $(S \rightarrow R)$, and relay-to-destination $(R \rightarrow D)$ links, respectively, and $\theta$ is the angle between lines $S \rightarrow R$ and $R \rightarrow D$. Assuming the path loss between $S \rightarrow D$ to be unity, the relative gain of $S \rightarrow R$ and $R \rightarrow D$ links are defined as $G_{S R}=\left(d_{S D} / d_{S R}\right)^{v}$ and $G_{R D}=\left(d_{S D} / d_{R D}\right)^{v}$, respectively, where $v$ is the pathloss coefficient [12]. We further define the relative geometrical gain $\mu=G_{S R} / G_{R D}$ (in decibels), which indicates the location of the relay with respect to the source and destination. The more negative this ratio (given in decibels) is, the more closely

\footnotetext{
${ }^{1}$ For the sake of presentation, in the rest of this paper, we use the term "Nakagami" instead of the actual term "Nakagami- $m$ " in a similar manner to [16].
}

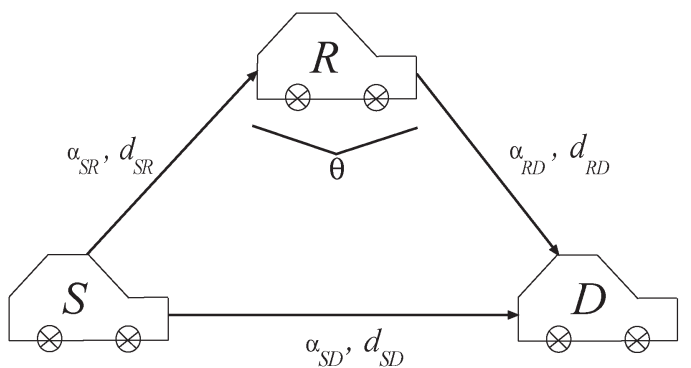

(a)

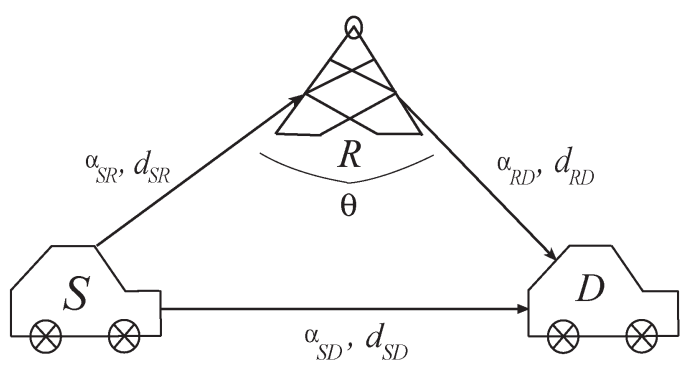

(b)

Fig. 1. (a) Vehicle-assisted V2V communication. (b) AP-assisted V2V communication.

the relay is placed to the destination node. On the other hand, positive values of this ratio indicate that the relay is more close to the source node. The particular case of $\mu=0 \mathrm{~dB}$ means that both source and destination nodes have the same distance to the relay.

We assume that all underlying channels are quasi-static, which is well justified for vehicular communication scenarios in rush-hour traffic. In Fig. 1(a), $\alpha_{S R}, \alpha_{R D}$, and $\alpha_{S D}$ represent $S \rightarrow R, R \rightarrow D$, and $S \rightarrow D$ links' complex fading coefficients whose magnitudes $h_{S R}, h_{R D}$, and $h_{S D}$, respectively, follow the cascaded Nakagami distribution for the vehicle-assisted scenario. These magnitudes are assumed to be the product of statistically independent, but not necessarily identically distributed, two Nakagami random variables [16]. Therefore, we have $h_{S D}=h_{S D_{1}} h_{S D_{2}}, h_{S R}=h_{S R_{1}} h_{S R_{2}}$, and $h_{R D}=h_{R D_{1}} h_{R D_{2}}$ for $S \rightarrow D, S \rightarrow R$, and $R \rightarrow D$ links, respectively, with the probability density function (pdf)

$$
f_{h}(h)=\frac{2}{h \Gamma\left(m_{1}\right) \Gamma\left(m_{2}\right)} G_{0,2}^{2,0}\left(\left.\frac{m_{1} m_{2} h^{2}}{\Omega_{1} \Omega_{2}}\right|_{m_{1}, m_{2}} ^{-}\right)
$$

where the subscripts $S D, S R$, and $R D$ are dropped for convenience. Here, $G_{0,2}^{2,0}(. \mid$ :) is the Meijer $G$-function, and $\Gamma($.$) is$ the Gamma function [17]. In (1), $m_{l}, l=1,2$, is a parameter describing the fading severity given by $m_{l}=\Omega_{l}^{2} / E\left[\left(h_{l}^{2}-\right.\right.$ $\left.\left.\Omega_{l}\right)^{2}\right] \geq 1 / 2$, with $\Omega_{l}=E\left[h_{l}^{2}\right]$ and $E[$.$] denoting the expecta-$ tion operator. Taking $\Omega_{l}=1$, one can normalize the power of the fading process to unity. Furthermore, note that the pdf in (1) reduces to the cascaded Rayleigh distribution when $m_{l}=1$ [18]. In the AP-assisted scenario illustrated in Fig. 1(b), the $S \rightarrow D$ link is still modeled as cascaded Nakagami; however, $S \rightarrow R$ and $R \rightarrow D$ links are now subject to Nakagami fading. This is justifiable considering that the relay node is an AP 
elevated well above street level. Under this scenario, the pdf for $h_{S R}$ and $h_{R D}$ is given by

$$
f_{h}(h)=\frac{2 m^{m}}{\Omega^{m} \Gamma(m)} h^{2 m-1} \exp \left(-\frac{m}{\Omega} h^{2}\right) .
$$

Note that the Nakagami distribution encloses both the Rayleigh and Rician distributions. For $m>1$, it closely approximates the Rician distribution, which is used to model fading channels with a line-of-sight (LOS) component. There is a one-to-one mapping between the $m$ parameter and the Rician factor, which can be written as $\sqrt{m^{2}-m} /\left(m-\sqrt{m^{2}-m}\right)$, $m \geq 1$ in terms of $m$ [19].

The transmission model under consideration builds upon the receive diversity cooperation protocol ${ }^{2}$ [7], [12]. This protocol effectively implements a single-input-multiple-output (SIMO) scheme in a distributed fashion, realizing receive diversity advantages. In the receive diversity protocol, the source node broadcasts to the relay and destination nodes over the first transmission phase. In the second transmission phase, only the relay node communicates with the destination node. Therefore, the signal transmitted both to the relay and destination nodes over the two transmission phases is the same. Let $x$ denote the transmitted signal chosen from an $M$-ary phase-shift keying (PSK) or $M$-ary quadratic-amplitude modulation (QAM) constellation. Considering path-loss effects, the received signals at the relay and destination are given as

$$
\begin{aligned}
r_{R} & =\sqrt{2 G_{S R} K E} h_{S R} x+n_{R} \\
r_{D 1} & =\sqrt{2 K E} h_{S D} x+n_{D 1}
\end{aligned}
$$

where $n_{R}$ and $n_{D_{1}}$ are the independent samples of zero-mean complex Gaussian random variables with variance $N_{0} / 2$ per dimension. Here, the total energy (to be used by both source and relay terminals) is $2 E$ during two time slots, yielding an average power in proportion to $E$ per time slot, i.e., assuming a unit time duration. $K$ is an optimization parameter that controls the fraction of power reserved for the broadcasting phase. Setting $K=1 / 2$ yields the EPA scheme.

The relay terminal normalizes the received signal $r_{R}$ by a factor of $\sqrt{E\left[\left|r_{R}\right|^{2}\right]}=\sqrt{2 G_{S R} K E+N_{0}}$ and retransmits the resulting signal during the second time slot. After proper normalization, the received signal at the destination is therefore given by [21]

$$
r_{D 2}=\sqrt{a E} h_{S R} h_{R D} x+n_{D 2}
$$

where $\quad a=\left(2 G_{S R} K\right) /\left(A+h_{R D}^{2}\right), \quad$ with $\quad A=[1+$ $\left.\left.2 G_{S R} K\left(E / N_{0}\right)\right] / 2 G_{R D}(1-K)\left(E / N_{0}\right)\right]$, and $n_{D_{2}}$ is a conditionally zero-mean complex Gaussian random variable with variance $N_{0} / 2$ per dimension. Writing (4) and (5) in a matrix notation, we have

$$
\underbrace{\left[\frac{r_{D 1}}{r_{D 2}}\right]^{T}}_{\mathbf{r}}=\underbrace{\left[\begin{array}{l}
h_{S D} \\
h_{S R} h_{R D}
\end{array}\right]^{T}}_{\mathbf{h}} \underbrace{\left[\begin{array}{cc}
\sqrt{2 K E} x & 0 \\
0 & \sqrt{a E x}
\end{array}\right]}_{\mathbf{x}}+\underbrace{\left[\begin{array}{l}
n_{D 1} \\
n_{D 2}
\end{array}\right]^{T}}_{\mathbf{n}} .
$$

\footnotetext{
${ }^{2}$ This is referred as orthogonal amplify-and-forward relaying in [20].
}

\section{PEP ANd Diversity GAin ANALysis}

In this section, we investigate the diversity order for the cooperative vehicular scheme under consideration through the derivation of the PEP. The PEP is the building block for the derivation of union bounds to the error rates, which will later be used as an objective function for OPA. We assume maximum-likelihood decoding with perfect knowledge of the channel state information at the receiver. A Chernoff bound on the conditional PEP is given by [22]

$$
P(\mathbf{X}, \hat{\mathbf{X}} \mid \mathbf{h}) \leq \exp \left(-\frac{d^{2}(\mathbf{X}, \hat{\mathbf{X}} \mid \mathbf{h})}{4 N_{0}}\right)
$$

where the Euclidean distance (conditioned on fading channel coefficients) between $\mathbf{X}$ and $\hat{\mathbf{X}}$ is $d^{2}(\mathbf{X}, \hat{\mathbf{X}} \mid \mathbf{h})=\mathbf{h}(\mathbf{X}-\hat{\mathbf{X}})$ $(\mathbf{X}-\hat{\mathbf{X}})^{H} \mathbf{h}^{H}$ Here, ()$^{H}$ denotes Hermitian transpose. Substituting $d^{2}(\mathbf{X}, \hat{\mathbf{X}} \mid \mathbf{h})$ in (7), we have

$$
P\left(\mathbf{X}, \hat{\mathbf{X}} \mid h_{S D}, h_{S R} h_{R D}\right) \leq \exp \left(-\frac{\beta}{2}\left(2 K h_{S D}^{2}+a h_{S R}^{2} h_{R D}^{2}\right)\right)
$$

where $\beta=|x-\hat{x}|^{2}\left(E / N_{0}\right) / 2$. Since the channel distributions differ for two scenarios under consideration, we present them separately in the following.

\section{A. PEP for the Vehicle-Assisted Scenario}

In this scenario, another vehicle in the vicinity of the source vehicle acts as a relay. Therefore, all underlying channels can be modeled as cascaded Nakagami fading. Let $m_{S D_{1}}$, $m_{S D_{2}}, m_{S R_{1}}, m_{S R_{2}}, m_{R D_{1}}$, and $m_{R D_{2}}$ denote the $m$ parameters of the Nakagami random variables representing the corresponding links. Let $y_{S D}, y_{S R}$, and $y_{R D}$ denote $y_{S D}=$ $h_{S D}^{2}, y_{S R}=h_{S R}^{2}$, and $y_{R D}=h_{R D}^{2}$, respectively, each with the normalized pdf

$$
f_{y}(y)=\frac{1}{y \Gamma\left(m_{1}\right) \Gamma\left(m_{2}\right)} G_{0,2}^{2,0}\left(\left.m_{1} m_{2} y\right|_{m_{1}, m_{2}} ^{-}\right)
$$

where the subscripts $S D, S R$ and $R D$ are dropped for convenience. Averaging (8) with respect to $y_{S D}$ and using the closedform solution [17, eq. (713.1)]

$$
\int_{0}^{\infty} z^{-\rho} \exp (-\gamma z) G_{0,2}^{2,0}\left(\left.\eta z\right|_{b_{1}, b_{2}} ^{-}\right) d z=\gamma^{\rho-1} G_{1,2}^{2,1}\left(\left.\frac{\eta}{\gamma}\right|_{b_{1}, b_{2}} ^{\rho}\right)
$$

for the resulting expression, we have

$$
P\left(\mathbf{X}, \hat{\mathbf{X}} \mid y_{S R}, y_{R D}\right) \leq \exp \left(-\frac{a \beta}{2} y_{S R} y_{R D}\right)
$$

$$
\times \frac{1}{\prod_{l=1}^{2} \Gamma\left(m_{S D_{l}}\right)} G_{1,2}^{2,1}\left(\left.\frac{m_{S D_{1}} m_{S D_{2}}}{K \beta}\right|_{m_{S D_{1}}, m_{S D_{2}}} ^{1}\right) .
$$


Further averaging (11) over $y_{S R}$ and again using (10), we obtain

$$
\begin{aligned}
& P\left(\mathbf{X}, \hat{\mathbf{X}} \mid y_{R D}\right) \leq G_{1,2}^{2,1}\left(\left.\frac{m_{S D_{1}} m_{S D_{2}}}{K \beta}\right|_{m_{S D_{1}}, m_{S D_{2}}} ^{1}\right) \\
& \times \frac{1}{\prod_{l=1}^{2} \Gamma\left(m_{S D_{l}}\right) \Gamma\left(m_{S R_{l}}\right)} G_{1,2}^{2,1}\left(\left.\frac{2 m_{S R_{1}} m_{S R_{2}}}{a \beta y_{R D}}\right|_{m_{S R_{1}}, m_{S R_{2}}} ^{1}\right) .
\end{aligned}
$$

Finally, averaging (12) over $y_{R D}$, we obtain an upper bound on the PEP as

$$
\begin{aligned}
P(\mathbf{X}, \hat{\mathbf{X}}) & \\
\leq & \frac{1}{\prod_{l=1}^{2} \Gamma\left(m_{S D_{l}}\right) \Gamma\left(m_{S R_{l}}\right) \Gamma\left(m_{R D_{l}}\right)} \\
& \times G_{1,2}^{2,1}\left(\left.\frac{m_{S D_{1}} m_{S D_{2}}}{K \beta}\right|_{m_{S D_{1}}, m_{S D_{2}}} ^{1}\right) \\
& \times \int_{0}^{\infty} y_{R D}^{-1} G_{1,2}^{2,1}\left(\left.\frac{m_{S R_{1}} m_{S R_{2}}}{K G_{S R} \beta}\left(1+\frac{A}{y_{R D}}\right)\right|_{m_{S R_{1}}, m_{S R_{2}}} ^{1}\right) \\
& \times G_{0,2}^{2,0}\left(\left.m_{R D_{1}} m_{R D_{2}} y_{R D}\right|_{m_{R D_{1}}, m_{R D_{2}}} ^{-}\right) d y_{R D} .
\end{aligned}
$$

To the best of our knowledge, a closed-form solution for (13) is unfortunately not available for the general case, yet this single integral can easily be numerically evaluated through commercially available mathematics software such as MATLAB, Mathematica, or Maple. Furthermore, for some certain relay locations, (13) can further be simplified. For example, consider the case when the relay is close to the destination (i.e., $\mu \ll 1$ ). Under sufficiently high signal-to-noise ratios (SNRs), it can be shown that (13) can be solved as

$$
\begin{aligned}
P(\mathbf{X}, \hat{\mathbf{X}}) \leq & G_{S R}^{-m_{S R_{1}}}\left[\prod_{l=1}^{2} m_{S R_{l}}\right]^{m_{S R_{1}}}\left[\prod_{l=1}^{2} m_{S D_{l}}\right]^{m_{S D_{1}}} \\
& \times U\left[m_{S D_{1}}, 1+m_{S D_{1}}-m_{S D_{2}}, \frac{m_{S D_{1}} m_{S D_{2}}}{K \beta}\right] \\
& \times U\left[m_{S R_{1}}, 1+m_{S R_{1}}-m_{S R_{2}}, \frac{m_{S R_{1}} m_{S R_{2}}}{K G_{S R} \beta}\right] \\
& \times(K \beta)^{-m_{S D_{1}}-m_{S R_{1}}}
\end{aligned}
$$

where $U(., .,$.$) is the hypergeometric U$-function [17]. From (14), one can check that this system achieves an asymptotical diversity order of $\min \left(m_{S D_{1}}, m_{S D_{2}}\right)+\min \left(m_{S R_{1}}, m_{S R_{2}}\right)$. It can be noted that the derived PEP in (14) includes cascaded Rayleigh fading as a special case. Inserting $m_{S D_{1}}=$ $m_{S D_{2}}=m_{S R_{1}}=m_{S R_{2}}=m_{R D_{1}}=m_{R D_{2}}=1$ in (14), we have $U(1,1,.) \rightarrow 1$, simplifying (14) to

$$
P(\mathbf{X}, \hat{\mathbf{X}}) \leq \frac{4 K^{-2}}{G_{S R}|x-\hat{x}|^{4}}\left(\frac{E}{N_{0}}\right)^{-2}
$$

which yields an asymptotic diversity order of two.

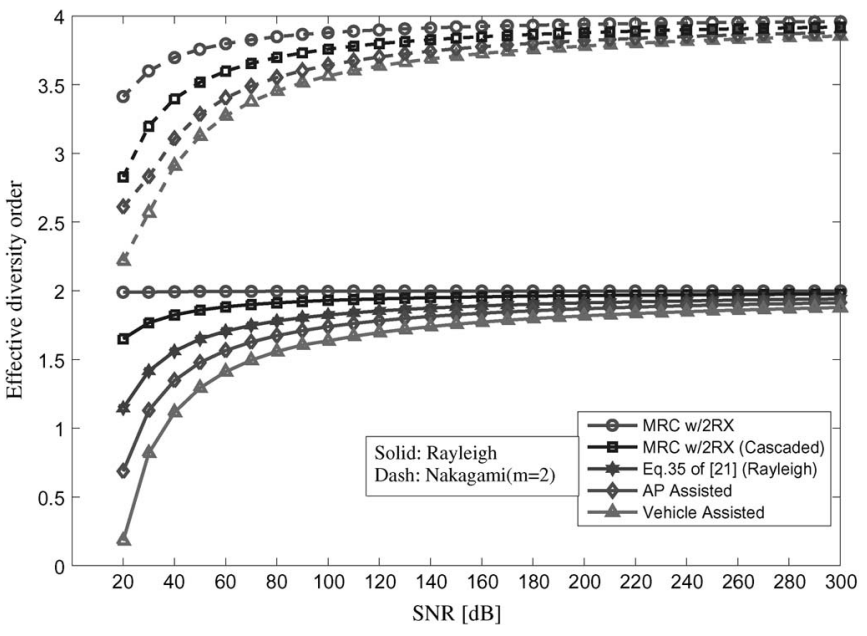

Fig. 2. Effective diversity order for the V2V system over conventional and cascaded Rayleigh and Nakagami $(m=2)$ fading processes.

\section{B. PEP for the AP-Assisted Scenario}

In the AP-assisted case, the channel between source and destination vehicles is modeled by cascaded Nakagami fading, whereas source-to-relay AP and relay AP-to-destination channels are modeled by Nakagami fading. Averaging (11) with respect to $h_{S R}$ and $h_{R D}$, which are now Nakagami distributed, we obtain

$$
\begin{aligned}
P(\mathbf{X}, \hat{\mathbf{X}}) \leq & \frac{1}{\prod_{l=1}^{2} \Gamma\left(m_{S D_{l}}\right) \Gamma\left(m_{S R_{l}}\right) \Gamma\left(m_{R D_{l}}\right)} \\
& \times G_{1,2}^{2,1}\left(\left.\frac{m_{S D_{1}} m_{S D_{2}}}{K \beta}\right|_{m_{S D_{1}}, m_{S D_{2}}} ^{1}\right) \\
& \times \int_{0}^{\infty} y_{R D}^{-1} G_{1,1}^{1,1}\left(\left.\frac{m_{S R}}{K G_{S R} \beta}\left(1+\frac{A}{y_{R D}}\right)\right|_{m_{S R}} ^{1}\right) \\
& \times G_{0,1}^{1,0}\left(\left.m_{R D} y_{R D}\right|_{m_{R D}} ^{-}\right) d y_{R D} .
\end{aligned}
$$

Under $\mu \ll 1$ and sufficiently high SNRs, we have

$$
\begin{aligned}
P(\mathbf{X}, \hat{\mathbf{X}}) \leq & \left(m_{S R}\right)^{m_{S R}} G_{S R}^{-m_{S R}}\left[\prod_{l=1}^{2} m_{S D_{l}}\right]^{m_{S D_{1}}} \\
& \times U\left[m_{S D_{1}}, 1+m_{S D_{1}}-m_{S D_{2}}, \frac{m_{S D_{1}} m_{S D_{2}}}{K \beta}\right] \\
& \times(K \beta)^{-m_{S D_{1}}-m_{S R}} .
\end{aligned}
$$

From (17), it can be shown that an asymptotic diversity order of $\min \left(m_{S D_{1}}, m_{S D_{2}}\right)+m_{S R}$ is available. Further note that the derived PEP in (17) includes cascaded Rayleigh fading as a special case. Inserting $m_{S D_{1}}=m_{S D_{2}}=m_{S R}=1$, (17) simplifies to (15), which yields an asymptotic diversity order of two.

To have a better understanding of how much diversity gain is achievable in various $\mathrm{SNR}=E / N_{0}$ ranges, we plot in Fig. 2 the effective (instantaneous) diversity order [18], which is simply the slope of the derived PEP as a function of the average SNR, i.e., $-\log P(\mathbf{X}, \hat{\mathbf{X}}) / \log \left(E / N_{0}\right)$. We consider the vehicle- and AP-assisted cases for both cascaded Nakagami and cascaded Rayleigh fading under consideration. 
As shown in Fig. 2, the asymptotical diversity orders for the vehicle-assisted scheme are 4 and 2, respectively, for cascaded Nakagami $(m=2)$ and cascaded Rayleigh fading. This confirms our earlier observation on the diversity order given by $\min \left(m_{S D_{1}}, m_{S D_{2}}\right)+\min \left(m_{S R_{1}}, m_{S R_{2}}\right)$. For the AP-assisted scheme, the diversity orders remain the same, which can be confirmed through $\min \left(m_{S D_{1}}, m_{S D_{2}}\right)+m_{S R}$.

As benchmarks, we include the performance of maximal ratio combining (MRC) with two colocated receive antennas over Nakagami $(m=2)$, cascaded Nakagami $(m=2)$, Rayleigh, and cascaded Rayleigh fading channels. It is obvious in Fig. 2 that for the MRC scheme, the effective diversity order converges to its asymptotical values of 4 and 2 over conventional Nakagami and Rayleigh fading, respectively. Convergence gets slower for the cascaded channels, and the asymptotical diversity order is observed for very high SNR values. Another benchmark is the performance of the receive diversity cooperative scheme under consideration over the Rayleigh fading channel. ${ }^{3}$ It is observed that the relaying link (i.e., the cascaded nature of the channel over $S \rightarrow R \rightarrow D$ link) further slows down the convergence. The performance of our vehicleassisted scheme suffers from both the presence of cascaded Nakagami (or Rayleigh) fading channels in the $S \rightarrow D$ link and the cascaded structure of two Nakagami (or Rayleigh) fading channels over the $S \rightarrow R \rightarrow D$ link. Therefore, the convergence of the diversity order to its asymptotical value becomes the slowest.

\section{Average PEP Over the Relay Location}

In this section, we investigate the average PEP for the vehicle-assisted scenario to take into account the vehicle relay's movement. Normalizing the distance between the source and the destination to unity (i.e., $d_{S D}=1$ ) and assuming $v=2$ and $\theta=\pi$, we have $\mu=G_{S R} / G_{R D}=\left(\left(1-d_{S R}\right) / d_{S R}\right)^{2}$, where $d_{S R}$ is the distance between the source and the relay. We also assume that $d_{S R}$ is modeled as a uniformly distributed random variable. The pdf of $d_{S R}$ is therefore given as $f\left(d_{S R}\right)=1$, $0 \leq d_{S R} \leq 1$. The pdf of $\mu$ can be then calculated as

$$
f(\mu)=\frac{1}{2 \sqrt{\mu}(1+\sqrt{\mu})^{2}}, \quad 0 \leq \mu \leq \infty .
$$

Rewriting (13) in terms of $\mu$, we obtain

$$
\begin{aligned}
P(\mathbf{X}, & \hat{\mathbf{X}}) \\
\leq & \frac{1}{\prod_{l=1}^{2} \Gamma\left(m_{S D_{l}}\right) \Gamma\left(m_{S R_{l}}\right) \Gamma\left(m_{R D_{l}}\right)} \\
& \times G_{1,2}^{2,1}\left(\left.\frac{m_{S D_{1}} m_{S D_{2}}}{K \beta}\right|_{m_{S D_{1}}, m_{S D_{2}}} ^{1}\right) \\
& \times \int_{0}^{\infty} y_{R D}^{-1} G_{1,2}^{2,1}\left(\left.\frac{m_{S R_{1}} m_{S R_{2}}}{K(1+\sqrt{\mu})^{2} \beta}\left(1+\frac{B}{y_{R D}}\right)\right|_{m_{S R_{1}}, m_{S R_{2}}} ^{1}\right. \\
& \times G_{0,2}^{2,0}\left(\left.m_{R D_{1}} m_{R D_{2}} y_{R D}\right|_{m_{R D_{1}}, m_{R D_{2}}} ^{-}\right) d y_{R D}
\end{aligned}
$$

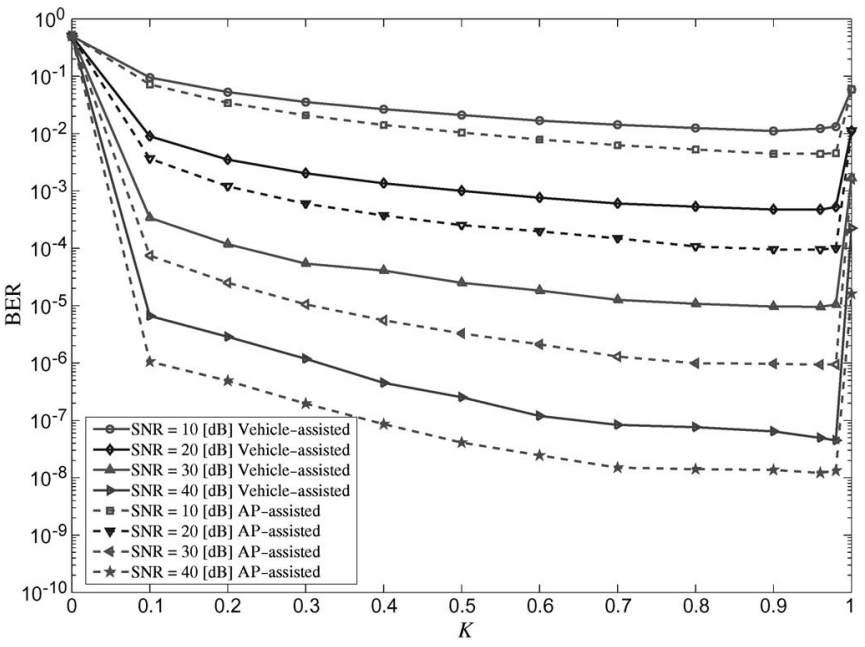

Fig. 3. BER versus $K(\mu=-30 \mathrm{~dB}$, 4-PSK, $\theta=\pi$, and $v=2)$.

where

$$
B=\frac{\left[1+2(1+\sqrt{\mu})^{2} K\left(E / N_{0}\right)\right]}{\left[2(1+1 / \sqrt{\mu})^{2}(1-K)\left(E / N_{0}\right)\right]} .
$$

The average PEP can then be calculated numerically by

$$
\overline{P(\mathbf{X}, \hat{\mathbf{X}})}=\int_{0}^{\infty} f(\mu) P(\mathbf{X}, \hat{\mathbf{X}}) d \mu .
$$

\section{BER-Optimized POWER Allocation}

Although EPA guarantees full asymptotical diversity, only partial diversity gains are exploited in the practical SNR ranges, as illustrated in Fig. 2. To further improve the performance, we aim to optimally allocate the power between broadcasting and relaying phases. For optimization of the power allocation, we consider the BER as our objective function. The derived PEP expressions constitute the building block for the derivation of BER bounds. Specifically, a union bound on the BER is given by [23]

$$
P_{b} \leq \frac{1}{n} \sum_{\mathbf{X}} p(\mathbf{X}) \sum_{\mathbf{X} \neq \hat{\mathbf{X}}} q(\mathbf{X}, \hat{\mathbf{X}}) P(\mathbf{X}, \hat{\mathbf{X}})
$$

where $n$ is the number of information bits per transmission, $p(\mathbf{X})$ is the probability that codeword $\mathbf{X}$ is transmitted, $q(\mathbf{X}, \hat{\mathbf{X}})$ is the number of information bit errors in choosing another codeword $\hat{\mathbf{X}}$ instead of the transmitted codeword, and $P(\mathbf{X}, \hat{\mathbf{X}})$ is the corresponding PEP. The specific form of BER bounds depends on the modulation scheme. Since PEPs are dependent on the Euclidean distance, we introduce the notation $f\left(\Delta=|x-\hat{x}|^{2}\right) \hat{=} P(\mathbf{X}, \hat{\mathbf{X}})$ to explicitly demonstrate this dependence. Union bounds on BER for binary PSK (BPSK), 4-PSK, 16-PSK, and 16-QAM can be then expressed as

$$
\begin{aligned}
P_{b, \text { BPSK }} & \leq f(\Delta=4) \\
P_{b, 4-\mathrm{PSK}} & \leq f(\Delta=2)+f(\Delta=4)
\end{aligned}
$$


TABLE I

OPA PARAMETERS $K$ FOR THE Vehicle-Assisted CASE (NAKAgAmi, $m=2$ )

\begin{tabular}{|c||c|c|c|c|c|c|c|c|c|c|c|c|}
\hline \multicolumn{1}{c||}{} & \multicolumn{3}{c|}{ BPSK } & \multicolumn{3}{c|}{ 4-PSK } & \multicolumn{3}{c|}{ 16-PSK } & \multicolumn{3}{c|}{$\begin{array}{c}\mu \\
\mu-Q A M \\
\mu\end{array}$} \\
$\mathrm{SNR}$ & $-30 \mathrm{~dB}$ & $0 \mathrm{~dB}$ & $30 \mathrm{~dB}$ & $-30 \mathrm{~dB}$ & $0 \mathrm{~dB}$ & $30 \mathrm{~dB}$ & $-30 \mathrm{~dB}$ & $0 \mathrm{~dB}$ & $30 \mathrm{~dB}$ & $-30 \mathrm{~dB}$ & $0 \mathrm{~dB}$ & $30 \mathrm{~dB}$ \\
{$[\mathrm{~dB}]$} & $K$ & $K$ & $K$ & $K$ & $K$ & $K$ & $K$ & $K$ & $K$ & $K$ & $K$ & $K$ \\
\hline 5 & 0.9745 & 0.6642 & 0.5271 & 0.9770 & 0.6686 & 0.5191 & 0.9694 & 0.6810 & 0.5211 & 0.9686 & 0.6820 & 0.5243 \\
10 & 0.9732 & 0.6632 & 0.5182 & 0.9780 & 0.6665 & 0.5142 & 0.9723 & 0.6805 & 0.5181 & 0.9730 & 0.6810 & 0.5162 \\
15 & 0.9730 & 0.6630 & 0.5092 & 0.9785 & 0.6664 & 0.5113 & 0.9732 & 0.6800 & 0.5162 & 0.9730 & 0.6790 & 0.5121 \\
20 & 0.9725 & 0.6624 & 0.5064 & 0.9785 & 0.6652 & 0.5054 & 0.9732 & 0.6790 & 0.5113 & 0.9735 & 0.6740 & 0.5084 \\
25 & 0.9715 & 0.6624 & 0.5041 & 0.9785 & 0.6652 & 0.5032 & 0.9732 & 0.6791 & 0.5086 & 0.9735 & 0.6730 & 0.5053 \\
30 & 0.9715 & 0.6624 & 0.5033 & 0.9785 & 0.6652 & 0.5021 & 0.9732 & 0.6791 & 0.5062 & 0.9735 & 0.6730 & 0.5032 \\
35 & 0.9715 & 0.6624 & 0.5027 & 0.9784 & 0.6652 & 0.5027 & 0.9740 & 0.6791 & 0.5052 & 0.9735 & 0.6730 & 0.5023 \\
40 & 0.9715 & 0.6624 & 0.5014 & 0.9784 & 0.6652 & 0.5014 & 0.9740 & 0.6780 & 0.5031 & 0.9735 & 0.6730 & 0.5022 \\
45 & 0.9715 & 0.6615 & 0.5017 & 0.9784 & 0.6652 & 0.5014 & 0.9740 & 0.6780 & 0.5020 & 0.9735 & 0.6730 & 0.5022 \\
\hline
\end{tabular}

TABLE II

OPA PARAMETERS $K$ FOR the AP-Assisted CASe (NAKAgAmi, $m=2$ )

\begin{tabular}{|c||c|c|c|c|c|c|c|c|c|c|c|c|}
\hline \multicolumn{1}{|c||}{} & \multicolumn{3}{c|}{ BPSK } & \multicolumn{3}{c|}{ 4-PSK } & \multicolumn{3}{c|}{$16-$ PSK } & \multicolumn{3}{c|}{$\begin{array}{c}\mu-\text { QAM } \\
\mu\end{array}$} \\
$\mathrm{SNR}$ & $-30 \mathrm{~dB}$ & $0 \mathrm{~dB}$ & $30 \mathrm{~dB}$ & $-30 \mathrm{~dB}$ & $0 \mathrm{~dB}$ & $30 \mathrm{~dB}$ & $-30 \mathrm{~dB}$ & $0 \mathrm{~dB}$ & $30 \mathrm{~dB}$ & $-30 \mathrm{~dB}$ & $0 \mathrm{~dB}$ & $30 \mathrm{~dB}$ \\
{$[\mathrm{~dB}]$} & $K$ & $K$ & $K$ & $K$ & $K$ & $K$ & $K$ & $K$ & $K$ & $K$ & $K$ & $K$ \\
\hline 5 & 0.9720 & 0.6267 & 0.5051 & 0.9752 & 0.6014 & 0.5180 & 0.9814 & 0.6260 & 0.5168 & 0.9844 & 0.6130 & 0.5163 \\
10 & 0.9706 & 0.6345 & 0.5034 & 0.9717 & 0.6235 & 0.5130 & 0.9806 & 0.6325 & 0.5161 & 0.9809 & 0.6315 & 0.5152 \\
15 & 0.9702 & 0.6526 & 0.5023 & 0.9705 & 0.6398 & 0.5120 & 0.9800 & 0.6460 & 0.5149 & 0.9732 & 0.6340 & 0.5131 \\
20 & 0.9700 & 0.6545 & 0.5041 & 0.9701 & 0.6538 & 0.5100 & 0.9729 & 0.6640 & 0.5047 & 0.9710 & 0.6350 & 0.5125 \\
25 & 0.9700 & 0.6572 & 0.5104 & 0.9700 & 0.6596 & 0.5080 & 0.9709 & 0.6683 & 0.5034 & 0.9703 & 0.6450 & 0.5080 \\
30 & 0.9700 & 0.6721 & 0.5082 & 0.9700 & 0.6714 & 0.5050 & 0.9703 & 0.6721 & 0.5032 & 0.9701 & 0.6583 & 0.5065 \\
35 & 0.9700 & 0.6735 & 0.5045 & 0.9700 & 0.6721 & 0.5040 & 0.9701 & 0.6730 & 0.5030 & 0.9700 & 0.6662 & 0.5053 \\
40 & 0.9700 & 0.6735 & 0.5028 & 0.9700 & 0.6721 & 0.5040 & 0.9700 & 0.6730 & 0.5020 & 0.9690 & 0.6680 & 0.5020 \\
45 & 0.9700 & 0.6736 & 0.5021 & 0.9700 & 0.6720 & 0.5030 & 0.9700 & 0.6730 & 0.5010 & 0.9690 & 0.6720 & 0.5010 \\
\hline
\end{tabular}

$$
\begin{aligned}
P_{b, 16-\mathrm{PSK}} \leq & 0.5 f(\Delta=0.1523)+f(\Delta=0.5858) \\
& +f(\Delta=1.2347)+f(\Delta=2) \\
& +1.25 f(\Delta=2.7655)+1.5 f(\Delta=3.4122) \\
& +1.25 f(\Delta=3.8479)+0.5 f(\Delta=4) \\
P_{b, 16 \mathrm{QAM}} \leq & 0.75 f(\Delta=0.4)+f(\Delta=1.6) \\
& +0.25 f(\Delta=3.6)+1.125 f(\Delta=0.8) \\
& +f(\Delta=3.2)+2.25 f(\Delta=2) \\
& +0.75 f(\Delta=4)+0.125 f(\Delta=7.2) \\
& +0.75 f(\Delta=5.2) .
\end{aligned}
$$

The resulting BER expressions need to be minimized with respect to the power-allocation parameter $K(0 \leq K \leq 1)$. It can readily be checked that these expressions are convex functions with respect to $K$. See, for example, Fig. 3, where we plot (23) with respect to $K$ for various values of SNR under vehicle- and AP-assisted scenarios in the case of Rayleigh fading. For this figure, we assume that $\mu=-30 \mathrm{~dB}, \theta=\pi$, and $v=2$.

The convexity of the functions under consideration guarantees that the local minimum found through optimization will indeed be a global minimum. Since no closed-form solution is available, we resort to numerical methods to solve this optimization problem. It should be emphasized that this problem need not be solved in real time for practical systems, because optimization does not depend on the instantaneous channel information or the input data. This means that the OPA values can be obtained a priori for given values of operating SNR and propagation parameters and can be stored for use as a lookup table in practical implementation.
TABLE III

OPA PARAMETERS $K$ FOR THE Vehicle-Assisted CASE With RANDOMLY DISTRIBUTED RELAY LOCATIONS (NAKAGAMI, $m=2$ )

\begin{tabular}{|c||c|c|c|c|}
\hline SNR[dB] & BPSK & 4-PSK & 16-PSK & 16-QAM \\
& $K$ & $K$ & $K$ & $K$ \\
\hline 5 & 0.6230 & 0.6410 & 0.6560 & 0.6340 \\
10 & 0.6420 & 0.6420 & 0.6630 & 0.6420 \\
15 & 0.6530 & 0.6530 & 0.6560 & 0.6520 \\
20 & 0.6620 & 0.6530 & 0.6560 & 0.6530 \\
25 & 0.6650 & 0.6670 & 0.6570 & 0.6540 \\
30 & 0.6640 & 0.6620 & 0.6570 & 0.6660 \\
35 & 0.6640 & 0.6620 & 0.6630 & 0.6650 \\
40 & 0.6640 & 0.6620 & 0.6630 & 0.6650 \\
45 & 0.6640 & 0.6620 & 0.6630 & 0.6550 \\
\hline
\end{tabular}

In Tables I and II, we present optimum values of $K$ for vehicle- and AP-assisted scenarios, respectively, for cascaded Nakagami $(m=2)$ fading. We assume that $\theta=\pi$ and $v=2$ and consider various values of relative geometrical gain $\mu=$ $G_{S R} / G_{R D}$ (in decibels). For $\mu=-30 \mathrm{~dB}$ (i.e., the relay is close to the destination), optimum values of $K$ approach 1 for both scenarios. Specifically, our results indicate that $\sim 97 \%$ of the overall power should be dedicated to the broadcasting phase. For $\mu=0 \mathrm{~dB}$ (i.e., the relay is located midway between the source and destination nodes), this percentage drops to $\sim 66 \%$ in the vehicle-assisted scenario. In the AP-assisted scenario, it changes in the range from $62 \%$ to $67 \%$ depending on the modulation type. For $\mu=30 \mathrm{~dB}$ (i.e., the relay is close to the source), $K$ is found to be $\sim 0.5$ for both scenarios, indicating that EPA is optimal. In Table III, we present optimum values of $K$ for the vehicle-assisted scenario based on the 


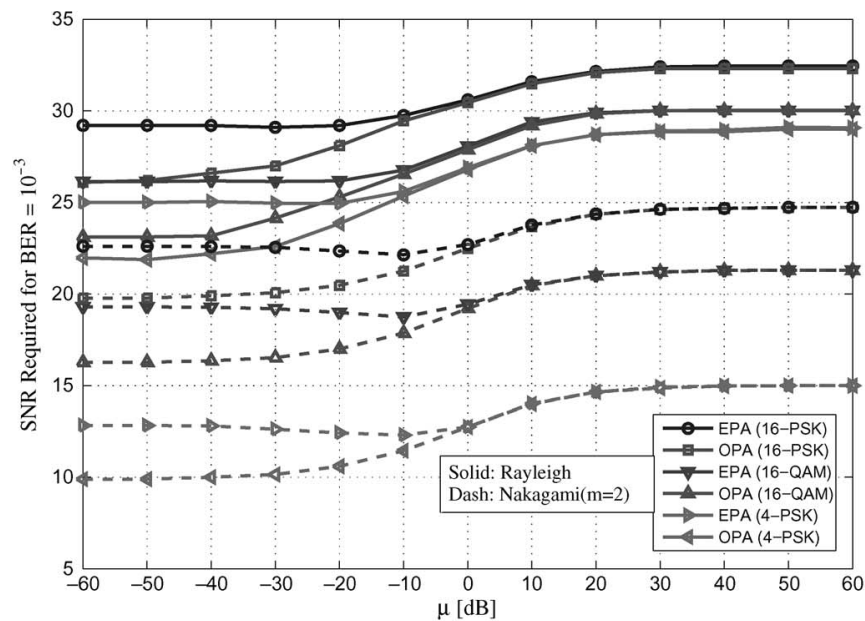

(a)

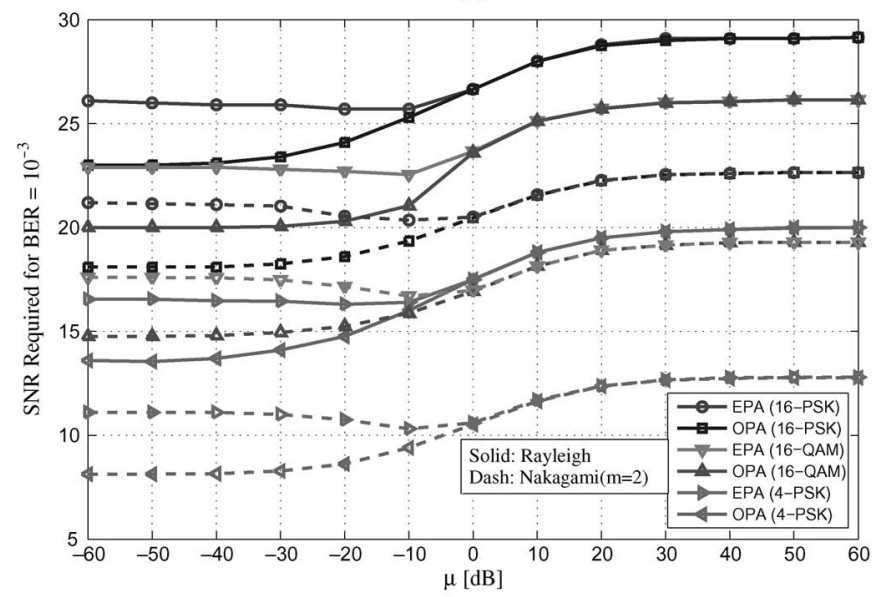

(b)

Fig. 4. (a) Power efficiency gains for a target BER of $10^{-3}$ for the vehicleassisted case ( $\theta=\pi$ and $v=2$ ). (b) Power efficiency gains for a target BER of $10^{-3}$ for the AP-assisted case $(\theta=\pi$, and $v=2)$.

average PEP, where $\mu$ follows the pdf given by (18). Our results show that $\sim 66 \%$ of the overall power should be dedicated to the broadcasting phase for all of the modulation types under consideration.

In Fig. 4, we demonstrate performance gains in power efficiency (as predicted by the derived union bounds) achieved by OPA over EPA for a target BER of $10^{-3}$ for vehicle- and APassisted cases. We assume 4-PSK, 16-PSK, and 16-QAM. In Fig. 4(a), for the vehicle-assisted scenario, we observe performance improvements of approximately $3 \mathrm{~dB}$ for all considered modulations, assuming negative values of $\mu$. For positive values of $\mu$, it is observed that OPA and EPA performance curves converge to each other, indicating the optimality of EPA for the Rayleigh and Nakagami $(m=2)$ cases. We also observe that EPA and OPA schemes require higher SNRs for 16-PSK and 16-QAM to provide the same performance of 4-PSK. As shown in Fig. 4(b), the AP-assisted scenario presents similar trends where OPA is more rewarding for relay locations near the destination. It can be also seen from these figures that lower SNR values are required when there exists an LOS component between the transmitters and receivers.

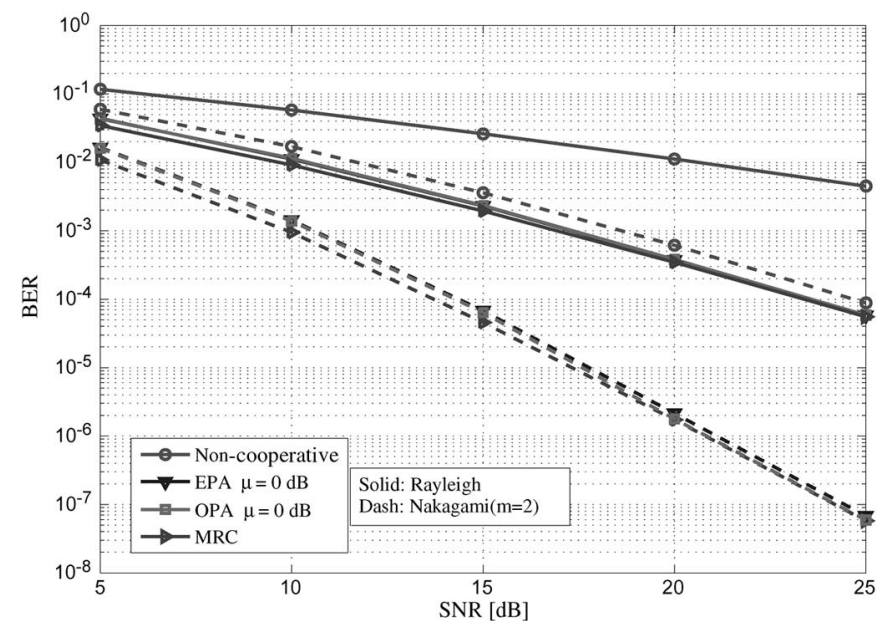

Fig. 5. BER performance results of the AP-assisted receive diversity protocol for $\mu=0 \mathrm{~dB}$ (4-PSK).

\section{Simulation Results And Discussion}

In this section, we present Monte Carlo simulation results to elaborate on our analytical results in the previous section. Error rate performances of the relay-assisted V2V scheme are simulated for two scenarios under consideration, assuming various relay locations and modulation schemes. In our simulations, for the AP-assisted scheme, the $S \rightarrow D$ link is modeled by cascaded Rayleigh/Nakagami $(m=2)$ fading, whereas $S \rightarrow R$ and $R \rightarrow D$ links are modeled by Rayleigh/Nakagami $(m=2)$. On the other hand, in the vehicle-assisted case, all links are modeled by cascaded Rayleigh/Nakagami $(m=2)$. We assume that $\theta=\pi$ and $v=2$ in our simulations.

In Fig. 5, we assume 4-PSK modulation for the cooperative scheme and consider a scenario where the relay AP is midway between source and destination vehicles (i.e., $\mu=0 \mathrm{~dB}$ ). Considering that two time slots are required to transmit one symbol in the considered cooperation (receive diversity) protocol, this modulation scheme achieves a throughput rate of $1 \mathrm{bit} / \mathrm{s} / \mathrm{Hz}$. The benchmark schemes are noncooperative direct transmission (i.e., no relaying) and MRC with two receive antennas. To maintain the same throughput rate with the cooperative scheme, they are simulated with BPSK.

As shown in Fig. 5, EPA and $\mathrm{OPA}^{4}$ BER performance results coincide, confirming our earlier observations through the PEP. We also observe in Fig. 5 that the performances of the cooperative scheme with EPA and OPA are 0.5 and $0.7 \mathrm{~dB}$ away from those of the MRC scheme at a BER of $10^{-3}$ for the Rayleigh and Nakagami cases, respectively. In Fig. 6, we present the frame-error-rate (FER) performance of the same schemes, where we assume frames of 260 data bits. Our results indicate that the FER performance is improved in a similar manner to the BER performance.

In Fig. 7, for $\mu=-30 \mathrm{~dB}$, we observe that the performance of the AP-assisted cooperative scheme improves over that obtained for $\mu=0 \mathrm{~dB}$. This is expected, because the cooperation protocol under consideration effectively realizes a

\footnotetext{
${ }^{4}$ Optimum power allocation values for the Rayleigh case can be found in [24].
} 


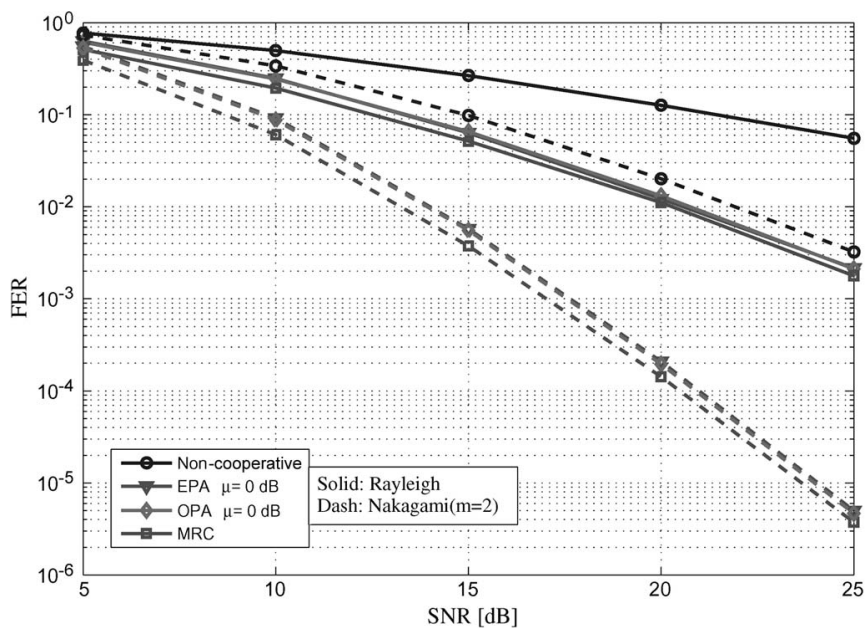

Fig. 6. FER performance results of the AP-assisted receive diversity protocol for $\mu=0 \mathrm{~dB}$ (4-PSK).

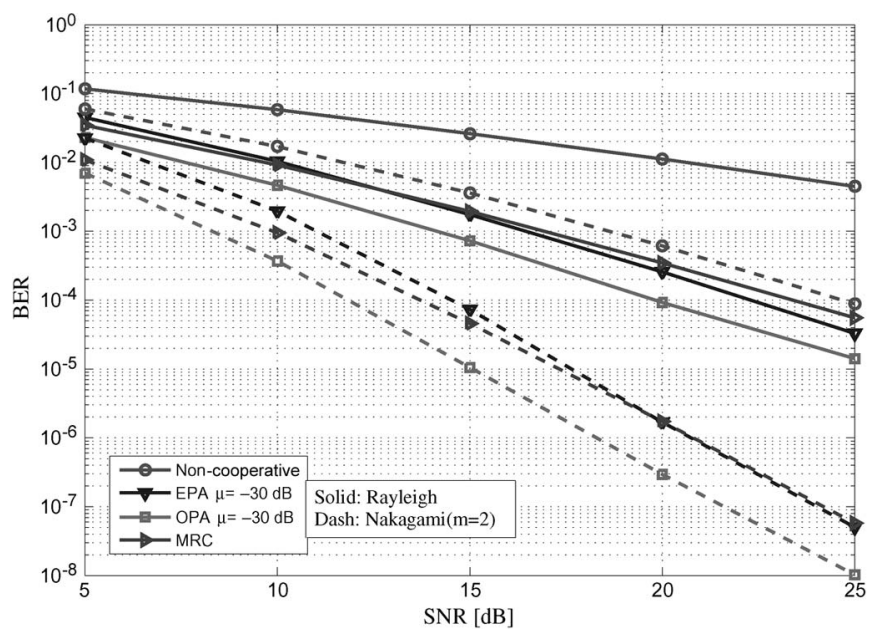

Fig. 7. BER performance results of the AP-assisted receive diversity protocol for $\mu=-30 \mathrm{~dB}$ (4-PSK).

distributed implementation of receive diversity; therefore, best performance results are obtained for cases where the relay is located near the destination. Particularly for OPA, we observe that the BER performance for $\mu=-30 \mathrm{~dB}$ improves by 2.8 and $2.5 \mathrm{~dB}$ compared with $\mu=0 \mathrm{~dB}$ at $\mathrm{BER}=10^{-3}$ for the Rayleigh and Nakagami cases, respectively. Furthermore, OPA provides an $\sim 3$-dB SNR gain with respect to EPA at a BER of $10^{-3}$, as expected from the power efficiency curves given in Fig. 4(b). The performance of the cooperative scheme with OPA outperforms the MRC scheme. It should be emphasized that the benchmarking MRC scheme is simulated over cascaded Rayleigh/Nakagami channels. Therefore, the cooperative scheme in the AP-assisted scenario can potentially outperform the MRC scheme, taking advantage of the underlying (more mild) Rayleigh/Nakagami links in $S \rightarrow R$ and $R \rightarrow D$ links, whereas two colocated receive antennas in the MRC scheme experience cascaded Rayleigh/Nakagami fading. ${ }^{5}$ As for FER

\footnotetext{
${ }^{5}$ In the vehicle-assisted scenario, the performance of MRC can be considered to be a lower bound on the performance of the cooperative scheme.
}

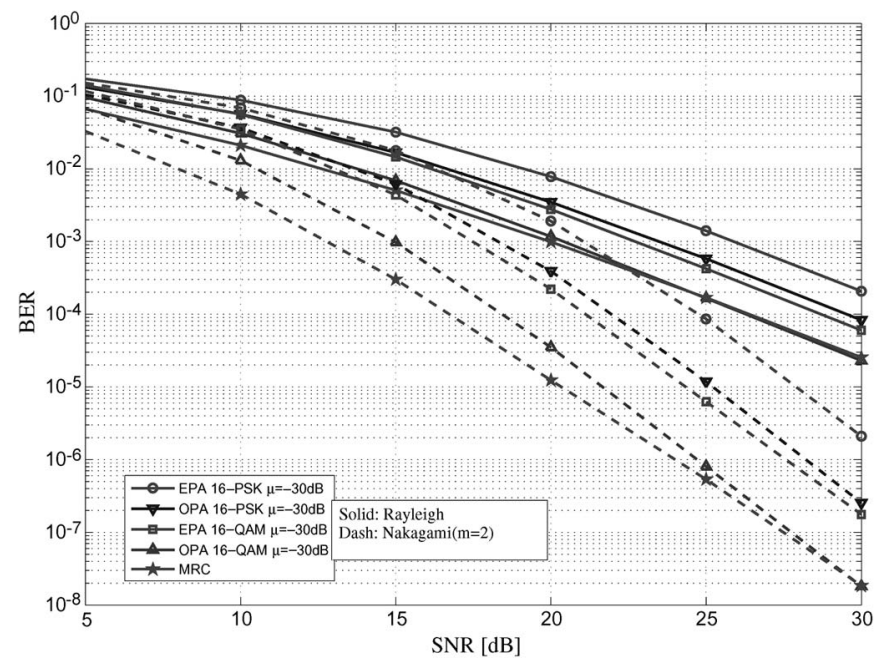

Fig. 8. BER performance results of the AP-assisted receive diversity protocol for $\mu=-30 \mathrm{~dB}$ (16-PSK and 16-QAM).

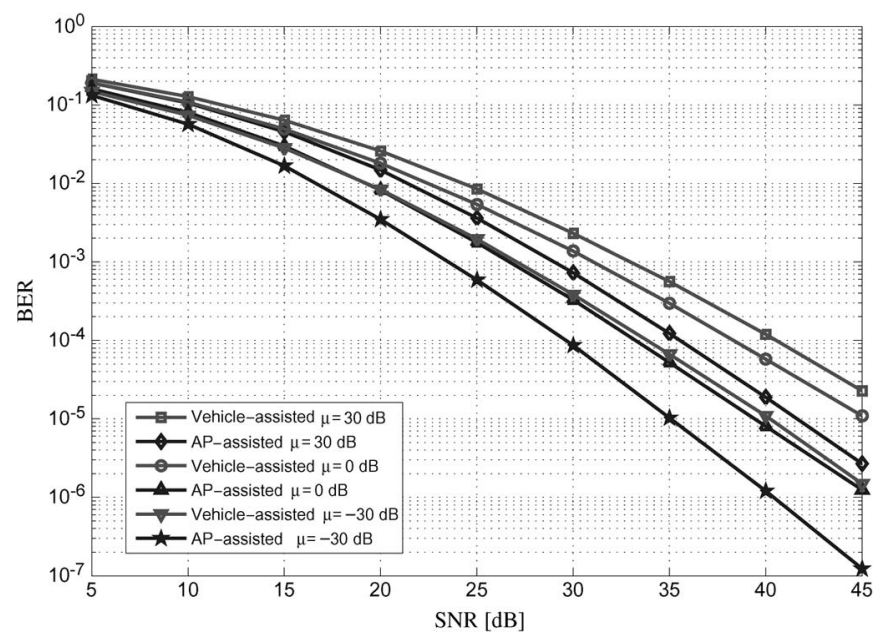

Fig. 9. Comparison of vehicle- and AP-assisted V2V cooperative schemes for various relay locations over cascaded Rayleigh fading channels (16-PSK, OPA).

performance, similar trends to the BER performance are observed. Those results are omitted due to space limitations.

In Fig. 8, we assume 16-PSK and 16-QAM for the APassisted cooperative scheme with $\mu=-30 \mathrm{~dB}$, whereas the benchmarking direct transmission and MRC schemes are simulated using 4-PSK. OPA provides $2.5-\mathrm{dB}$ power savings and outperforms the EPA counterpart at a BER of $10^{-3}$ for both 16-PSK and 16-QAM. However, even with OPA, performance curves are still about 0.5 and $2.2 \mathrm{~dB}$ away from the MRC bounds for the Rayleigh and Nakagami cases, respectively, under the assumption of 16-QAM. This is rather as a result of the crowded nature of signal constellations under consideration.

In Fig. 9, we compare the performance of AP- and vehicleassisted V2V cooperative schemes for $\mu=-30,0$, and $30 \mathrm{~dB}$, assuming 16-PSK. The optimum $K$ values for the Rayleigh case can be found in [25] for the vehicle-assisted scenario. We observe that the AP-assisted V2V scheme provides better performance than the vehicle-assisted counterpart since the APassisted scheme takes advantage of the underlying (more mild) 


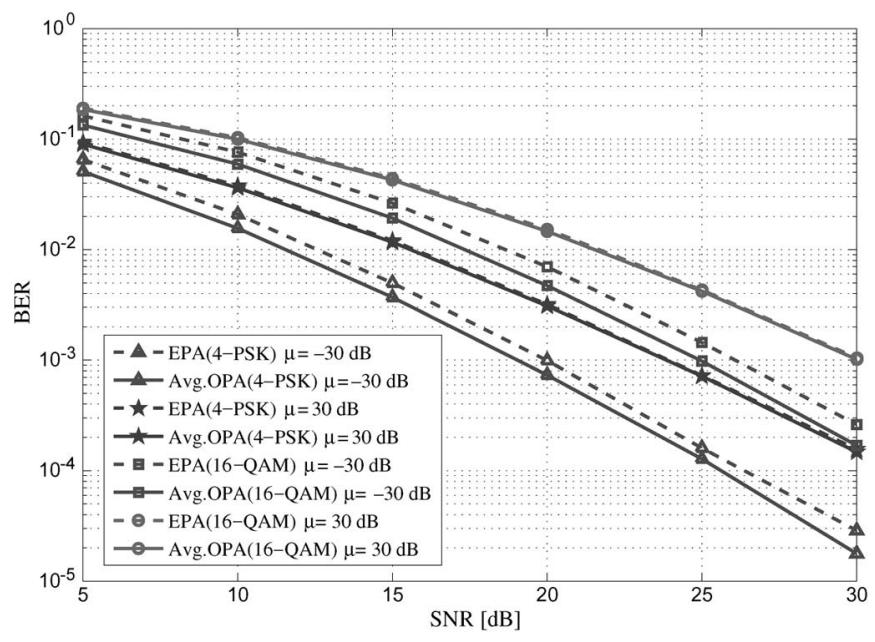

Fig. 10. BER performance results of the vehicle-assisted cooperative scheme optimized based on the average PEP.

TABLE IV

OPA PARAMETERS $K$ FOR THE Vehicle-Assisted CASE With RANDOMLY DisTRIBUTED RELAY LOCATIONS (RAYLEIGH)

\begin{tabular}{|c||c|c|c|c|}
\hline SNR[dB] & BPSK & 4-PSK & 16-PSK & 16-QAM \\
& $K$ & $K$ & $K$ & $K$ \\
\hline 5 & 0.6600 & 0.6700 & 0.6900 & 0.7100 \\
10 & 0.6500 & 0.6400 & 0.6800 & 0.6900 \\
15 & 0.6400 & 0.6400 & 0.6700 & 0.6800 \\
20 & 0.6300 & 0.6300 & 0.6600 & 0.6800 \\
25 & 0.6200 & 0.6300 & 0.6500 & 0.6700 \\
30 & 0.6200 & 0.6300 & 0.6500 & 0.6600 \\
35 & 0.6200 & 0.6300 & 0.6500 & 0.6600 \\
40 & 0.6200 & 0.6300 & 0.6500 & 0.6600 \\
45 & 0.6200 & 0.6300 & 0.6500 & 0.6600 \\
\hline
\end{tabular}

Rayleigh links in $S \rightarrow R$ and $R \rightarrow D$ links. Specifically, at $\mathrm{BER}=10^{-3}$, the AP-assisted scheme outperforms the vehicleassisted scheme by $4.1 \mathrm{~dB}$ for $\mu=30 \mathrm{~dB}$. The performance gap decreases to $3.5 \mathrm{~dB}$ for $\mu=-30 \mathrm{~dB}$.

In Fig. 10, we present the BER performance of the vehicleassisted V2V scheme optimized based on the average PEP given by (20) for the cascaded Rayleigh fading channel. The corresponding optimum $K$ values can be found in Table IV. We consider both 4-PSK and 16-QAM. Although no gain is observed for $\mu=30 \mathrm{~dB}$, approximately $1.4 \mathrm{~dB}$ is obtained with respect to the EPA case for $\mu=-30 \mathrm{~dB}$ at a BER of $10^{-3}$.

\section{CONCLUSION}

In this paper, we have analyzed and optimized the performance of a relay-assisted intervehicular scheme over cascaded Nakagami fading. For the single-relay scenario under consideration, through the derivation of the PEP, we have determined the effective diversity order and the maximum asymptotical diversity order. We have further formulated a power-allocation problem and determined the OPA values in the sense of minimizing the BER. Our simulation results indicate significant performance improvements, particularly for cases when the relay is located close to the destination.

\section{REFERENCES}

[1] K. Farkas, L. Ittode, J. Heidemann, T. Kosch, M. Strassberger, K. Laberteaux, L. Caminiti, D. Caveney, and H. Hada, "Vehicular communication," Pervasive Comput., vol. 5, no. 4, pp. 55-62, Oct./Dec. 2006.

[2] S. Biswas, R. Tatchikou, and F. Dion, "Vehicle-to-vehicle wireless communication protocols for enhancing highway traffic safety," IEEE Commun. Mag., vol. 44, no. 1, pp. 74-82, Jan. 2006.

[3] M. L. Sichitiu and M. Kihl, "Inter-vehicle communication systems: A survey," Commun. Surveys Tuts., vol. 10, no. 2, pp. 88-105, 2nd Quarter 2008.

[4] Y. Zang, L. Stibor, G. R. Hiertz, and H. J. Reumerman, "Vehicular wireless media network (VWMN)—A distributed broadband MAC for intervehicle communication," in Proc. 2nd ACM Workshop VANET, Cologne, Germany, Sep. 2005, pp. 95-96.

[5] P. Buccio, E. Masala, N. Kawaguchi, K. Takeda, and J. C. De Martin, "Performance evaluation of H. 264 video streaming over intervehicular 802.11 ad hoc networks," in Proc. IEEE PIMRC, Sep. 2005, pp. 1936-1940.

[6] J. Ott and D. Kutscher, "The drive-thru architecture: WLAN-based Internet access on the road," in Proc. IEEE VTC-Spring, Milan, Italy, May 2004, pp. 2615-2622.

[7] J. N. Laneman and G. W. Wornell, "Distributed space-time coded protocols for exploiting cooperative diversity in wireless networks," IEEE Trans. Inf. Theory, vol. 49, no. 10, pp. 2415-2525, Oct. 2003.

[8] A. Sendonaris, E. Erkip, and B. Aazhang, "User cooperation diversity. Part I: System description," IEEE Trans. Commun., vol. 51, no. 11, pp. 1927-1938, Nov. 2003.

[9] G. K. Karagiannidis, T. A. Tsiftsis, and R. K. Mallik, "Bounds of multihop relayed communications in Nakagami- $m$ fading," IEEE Trans. Commun., vol. 54, no. 1, pp. 18-22, Jan. 2006.

[10] T. A. Tsiftsis, "Performance of wireless multihop communications systems with cooperative diversity over fading channels," Int. J. Commun. Syst., vol. 20, no. 12, pp. 559-565, Dec. 2007.

[11] S. Yiu, R. Schober, and L. Lampe, "Decentralized distributed space-time trellis coding," IEEE Trans. Wireless Commun., vol. 6, no. 11, pp. 39853993, Nov. 2007.

[12] H. Ochiai, P. Mitran, and V. Tarokh, "Variable-rate two-phase collaborative communication protocols for wireless networks," IEEE Trans. Inf. Theory, vol. 52, no. 9, pp. 4299-4313, Sep. 2006.

[13] A. S. Akki and F. Haber, "A statistical model of mobile-to-mobile land communication channel," IEEE Trans. Veh. Technol., vol. 35, no. 1, pp. 2-7, Feb. 1986.

[14] I. Z. Kovacs, "Radio channel characterisation for private mobile radio systems: Mobile-to-mobile radio link investigations," Ph.D. dissertation, Aalborg Univ., Aalborg, Denmark, Sep. 2002.

[15] G. K. Karagiannidis, T. A. Tsiftsis, and N. C. Sagias, "A closedform upper-bound for the distribution of the weighted sum of Rayleigh variates," IEEE Commun. Lett., vol. 9, no. 7, pp. 589-591, Jul. 2005.

[16] G. K. Karagiannidis, N. C. Sagias, and P. T. Mathiopoulos, " $N^{*}$ Nakagami: A novel stochastic model for cascaded fading channels," IEEE Trans. Commun., vol. 55, no. 8, pp. 1453-1458, Aug. 2007.

[17] I. S. Gradshteyn and I. M. Ryzhik, Table of Integrals, Series and Products, 5th ed. New York: Academic, 1994.

[18] M. Uysal, "Diversity analysis of space-time coding in cascaded Rayleigh fading channels," IEEE Commun. Lett., vol. 10, no. 3, pp. 165-167, Mar. 2006.

[19] M. K. Simon and M. S. Alouini, Digital Communication Over Fading Channels, 2nd ed. Hoboken, NJ: Wiley, Jan. 2005.

[20] K. Azarian, H. El Gamal, and P. Schniter, "On the achievable diversity-multiplexing tradeoff in half-duplex cooperative channels," IEEE Trans. Inf. Theory, vol. 51, no. 12, pp. 4152-4172, Dec. 2005.

[21] M. M. Fareed and M. Uysal, "BER-optimized power allocation for fading relay channels," IEEE Trans. Commun., vol. 7, no. 6, pp. 2350-2359, Jun. 2008.

[22] V. Tarokh, N. Seshadri, and A. Calderbank, "Space-time codes for high data rate wireless communication: Performance criterion and code construction," IEEE Trans. Inf. Theory, vol. 44, no. 2, pp. 744-765, Mar. 1998.

[23] S. Benedetto and E. Biglieri, Principles of Digital Transmission With Wireless Applications. New York: Kluwer, 1999.

[24] H. İlhan, İ. Altunbaş, and M. Uysal, "Cooperative diversity for relayassisted inter-vehicular communication," in Proc. IEEE VTC-Spring, Singapore, May 2008, pp. 605-609.

[25] H. Illhan, İ. Altunbaş, and M. Uysal, "Optimized amplify-and-forward relaying for vehicular ad-hoc networks," in Proc. IEEE VTC - Fall, Calgary, AB, Canada, Sep. 2008, pp. 1-5. 


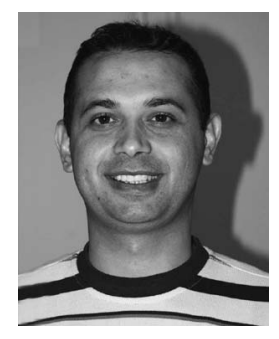

Hacı İlhan (S'07) was born in Turkey on September 16, 1976. He received the B.S. degree in electronics and communication engineering from Yildiz Technical University, Istanbul, Turkey, in 1999 and the M.Sc. degree in electronics and communication engineering from Istanbul Technical University in 2003 . He is currently working toward the Ph.D. degree in telecommunication engineering with Istanbul Technical University.

He is currently a Research and Teaching Assistant with the Department of Telecommunication, Istanbul Technical University. His general research interests are wireless communications. His specific areas of interest include distributed space-time coding, cooperative diversity, and power allocation for fading relay channels.

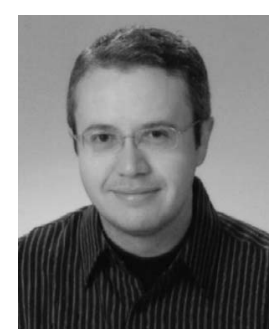

Murat Uysal (SM'07) was born in Istanbul, Turkey, in 1973. He received the B.Sc. and M.Sc. degrees in electronics and communication engineering from Istanbul Technical University in 1995 and 1998, respectively, and the Ph.D. degree in electrical engineering from Texas A\&M University, College Station, in 2001.

From 1995 to 1998 , he was a Research and Teaching Assistant with the Communication Theory Group, Istanbul Technical University. From 1998 to 2002 , he was with the Wireless Communication Laboratory, Texas A\&M University. During the fall of 2000, he was a Research Intern with AT\&T Labs-Research, Florham Park, NJ. Since 2002, he has been with the Department of Electrical and Computer Engineering, University of Waterloo, Waterloo, ON, Canada, where he is currently an Associate Professor. His general research interests are in the area of communications theory, with particular emphasis on wireless applications. His specific areas of interest include multiple-input-multiple-output communications, space-time coding, diversity techniques and coding for fading channels, cooperative transmission, performance analysis over fading channels, channel estimation and equalization, orthogonal frequency-division multiplexing, and free-space optical communication.

Dr. Uysal is an Associate Editor of the IEEE TRANSACTIONS ON WIRELESS COMmUnicAtions and the IEEE COMmUniCATIONS LETTERS. He served as a Guest Coeditor of the journal Wireless Communications and Mobile Computing Special Issue on "MIMO Communications" (published in 2004) and of the IEEE Journal on Selected AREas in Communications Special Issue on "Optical Wireless Communications" (to be published in 2010). Over the years, he has served on the Technical Program Committees of more than 50 international conferences in the communications area. He recently cochaired the IEEE International Conference on Communications (ICC'07) Communication Theory Symposium and chaired the IEEE Canadian Conference on Electrical and Computer Engineering (CCECE'08) Communications and Networking Symposium.

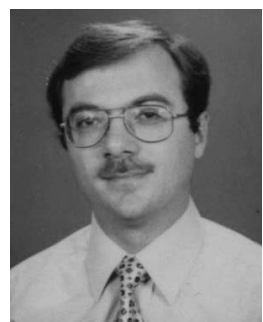

İbrahim Altunbaş was born in Sütçüler, Turkey, in 1967. He received the B.S., M.S., and Ph.D. degrees, from Istanbul Technical University, Istanbul, Turkey, in 1988,1992 , and 1999 , respectively, all in electrical engineering.

He was a Research Assistant from 1992 to 1999 and an Assistant Professor from 1999 to 2006 with Istanbul Technical University, where he is currently an Associate Professor. From January 2001 to November 2001, he was a Visiting Researcher with Texas A\&M University, College Station, and from November 2001 to September 2002, he was a Postdoctoral Fellow with the University of Ottawa, Ottawa, ON, Canada. His research interests include digital communications, information theory, error control coding and modulation, turbo coding, iterative decoding, multiple-input-multiple-output systems, space-time coding, and cooperative communication. 